\title{
Diagnostic Model for Sensitization in Workers Exposed to Occupational High Molecular Weight Allergens
}

\author{
Eva Suarthana, MD, MSc, ${ }^{1,2,3,4}$ Yvonne Vergouwe, $\mathrm{PhD}^{5}{ }^{5}$ Mark Nieuwenhuijsen, $\mathrm{PhD}^{6}$ \\ Dick Heederik, $\mathrm{PhD},{ }^{1}$ Diederick E. Grobbee, MD, $\mathrm{PhD}^{2}{ }^{2}$ and Evert Meijer, $\mathrm{MD}, \mathrm{PhD}^{1}{ }^{1}$
}

\begin{abstract}
Background Occupational allergy has great impact on workers exposed to high molecular weight (HMW) allergens. The present study is aimed to develop and validate a generic diagnostic model for sensitization to HMW allergens, defined as positive IgE.

Methods The model was developed in pooled data from Dutch laboratory animal (LA) workers and bakers using logistic regression analysis. Validity was assessed internally by bootstrapping procedure, and externally in British LA workers.

Results The model included working hours/week, work-related symptoms, total IgE, and $I g E$ to common allergen. Significant interactions between the type of work and the predictors resulted in different scores for LA workers and bakers. Internal and external validation showed that the model was satisfactorily calibrated and discriminated between workers at high and low risk of being sensitized.

Conclusions It is possible to develop a generic model for sensitization to occupational $H M W$ allergens. However, the weighing of predictors differs across specific work environments. Am. J. Ind. Med. 48:168-174, 2005. ๑ 2005 Wiley-Liss, Inc.
\end{abstract}

KEY WORDS: diagnostic research; occupational allergy; bakers; laboratory animal workers; validity

\section{INTRODUCTION}

Occupational allergy has long been recognized as an important problem in workers exposed to high molecular

${ }^{1}$ IRAS (Institute for Risk Assessment Sciences), Environmental and Occupational Health Group, Utrecht University. Utrecht, The Netherlands

${ }^{2}$ Julius Center for Health Sciences and Primary Care, University Medical Center Utrecht, Utrecht, The Netherlands

${ }^{3}$ Netherlands Institute of Health Sciences, Erasmus MC, Rotterdam, The Netherlands

${ }^{4}$ Epidemiology Post-graduate Program, Faculty of Public Health, University of Indonesia, Jakarta Pusat, Indonesia

${ }^{5}$ Department of Public Health, Erasmus MC, Rotterdam, The Netherlands

${ }^{6}$ Department of Occupational and Environmental Medicine, Imperial College (NHLI), London, United Kingdom

${ }^{\star}$ Correspondence to: Evert Meijer, IRAS (Institute for Risk Assessment Sciences), Environmental and Occupational Health Group, Utrecht University, P0 Box 80176, 3508 TD, Utrecht, The Netherlands.E-mail:E.Meijer@iras.uu.nl

Accepted 24 May 2005

DOI 10.1002/ajim.20199. Published online in Wiley InterScience

(www.interscience.wiley.com) weight (HMW) proteins that may cause allergic sensitization through IgE-mediated mechanisms. This particularly concerns rat urinary aeroallergen (RUA) and mouse urinary aeroallergen (MUA) exposure in laboratory animal (LA) workers, and wheat flour and fungal $\alpha$-amylase exposure in bakers [Houba et al., 1998a; Jeffrey et al., 1999; Nieuwenhuijsen et al., 1999, 2003].

The prevalence of occupational allergy has been reported to vary between $11 \%$ and $44 \%$ in LA workers and $5 \%$ and 30\% in bakers [Hollander et al., 1996; Houba et al., 1998a]. Work-related allergic symptoms, for example, asthma, rhinitis, conjunctivitis, and/or eczema are frequently severe enough to produce social and economic consequences at the individual level. A survey in the UK among patients diagnosed with asthma showed that persons with occupational asthma had greater difficulty in finding new jobs, greater loss of income, and were less likely to be currently employed than those with asthma that was unrelated to work [Houba et al., 1998a]. 
Exposure response studies show that, at the moment, sensitization can still occur when exposure is at the very lowest technologically achievable level [Houba et al., 1998a]. This stresses the importance of early detection of allergic sensitization in individual workers. However, standardized allergen preparations to demonstrate workrelated sensitization can be costly and may not always be available. Therefore, a simple and cheap diagnostic strategy, for example, a diagnostic decision model, is needed to identify workers at high risk of being sensitized to occupational HMW allergens.

A strategy for health surveillance in LA workers exposed to RUA and MUA was recently developed [Meijer et al., 2004] from 351 Dutch LA workers participating in an ongoing cohort study. However, it would be an advantage for occupational physicians if they have a generic diagnostic model for sensitization to HMW allergens, which they can apply in workers exposed to a broader spectrum HMW allergen. Therefore, the current study is aimed to develop and validate a single diagnostic model for sensitization to a broader spectrum occupational HMW allergens from questionnaires and laboratory tests, commonly used in occupational health practice. We, therefore, developed a diagnostic model in pooled data set of Dutch LA workers and bakers and validated the model in British LA workers.

In contrast to the former study, we use positive IgE test (class I) to HMW allergen to diagnose sensitization. This test was used rather than skin prick test (SPT), since within the European Union it is not allowed to prepare crude extracts of working place allergens to be used for SPT.

\section{MATERIALS AND METHODS}

\section{Study Design and Population}

The study population was drawn from LA facilities in the Netherlands and United Kingdom and bakeries in the Netherlands. Data of the Dutch bakers were derived from 21 Dutch bakeries [Houba et al., 1998b]. Data of Dutch LA workers were collected from a cohort of four universities, three commercial or industrial laboratories, and one laboratory school participated [Hollander et al., 1996]. LA facilities in the United Kingdom involved three institutions (two commercial and one academic) specializing in small animal research [Cullinan et al., 1994a]. The participation rates were $75 \%, 77 \%$, and $88 \%$, which resulted in 427 Dutch bakers, 579 Dutch LA workers, and 357 British LA workers, respectively.

The number of observations can differ from previously published analyses because only workers, from whom questionnaires and specific IgE results were available, were included. The total study population contained 343 Dutch bakers, 568 Dutch LA workers, and 352 British LA workers.

\section{Questionnaire}

Workers completed a short self-administered respiratory questionnaire, which was extended with questions on personal history of allergic symptoms to common allergens, symptoms suggesting airways hyper-responsiveness (AHR), work-related allergic symptoms, smoking habits, and job histories [Cullinan et al., 1994a; Hollander et al., 1996; Houba et al., 1998b].

A history of allergy ('Are you or have you been allergic to one or more agents?'), was considered present if they reported history of chest tightness, running nose or sneezing, running or itching eyes, and itching skin to common allergens such as house dust, domestic animals, food, or pollen. Persons were considered to have workrelated allergic symptoms if they give a positive answer to the question 'Do you have symptoms of allergy (e.g., chest tightness, running nose or sneezing, running or itching eyes, and itching skin) during working hours, after contact with a certain agent (e.g., flour in the bakery, rat in the laboratory) at work?'. Current smokers were defined as those who smoked more than one cigarette a day for at least one year.

Data about AHR and personal history of respiratory symptoms from Dutch and British data sets could not be used because they were collected using different questionnaires.

\section{Serologic Tests}

Specific IgE antibodies were measured in the blood serum. Specific IgE antibodies to wheat flour were measured with a commercial immunoassay (AlaSTAT; DPC, Apeldoorn, The Netherlands). Specific IgE antibodies to $\alpha$ amylase were measured with modified enzyme immunoassay (EIA) [Doekes et al., 1996]. Specific IgE antibodies to RUA and MUA were analyzed with CAP-RAST [Heederik et al., 1999]. Sera of class 1 or higher $(>0.35 \mathrm{kU} / \mathrm{L})$ were considered positive. Specific IgE antibodies to common allergens (house dust mites, grass pollen, birch pollen, cat fur, and dog fur) were measured with assays developed at the University of Wageningen, The Netherlands [Doekes et al., 1996]. An optical density (OD) of 492 exceeding the $\mathrm{OD}+0.05$ of the reagent blank (no serum control) was considered as a positive reaction. Total IgE was measured with a sandwich EIA, a level $\geq 100 \mathrm{kU} / \mathrm{L}$ was defined positive [Doekes et al., 1996].

\section{Outcome Definition}

Sensitization to HMW allergens was defined as class I positive IgE serology to wheat flour or $\alpha$-amylase allergen in bakers, and RUA or MUA in LA workers. 


\section{Data Analysis}

A diagnostic model for HMW allergens sensitization was developed in the pooled data of Dutch bakers and LA workers ( $\mathrm{n}=911$, the development set). The univariable and multivariable associations between workers' characteristics and presence of sensitization were modeled with logistic regression analysis. The regression coefficients are equal to the antilog of the odds ratios (OR).

We examined whether continuous variables could be transformed into simple categorizations. Cut-off values for the categories were chosen based on fitted restricted cubic spline (RCS) function [Harrell et al., 1996]. Model $\chi^{2}$ of the models with the continuous and categorized variables were compared to see whether the categorizations were reasonable.

We first considered a full model including potential diagnostic characteristics from the questionnaire and serologic test with univariable $P$ - values below 0.5 . Secondly, we used a backward stepwise procedure $(P<0.157)$ to select a subset of the strongest predictors of sensitization [Harrell et al., 1996; Steyerberg et al., 2000]. We also assessed whether the type of work (bakers or LA workers) modified the association of the selected predictors and sensitization using the interaction term. We performed an overall test to study the statistical significance of the interactions at $P=0.3$, conform current statistical guidelines [Harrell Frank, 2001].

Calibration of the model, the agreement between predicted probabilities of being sensitized and observed frequencies of sensitized workers, was assessed with the Hosmer-Lemeshow goodness-of-fit test. The discriminative ability was determined with the area under the receiver operating characteristic (ROC) curve. The ROC curve shows the relation between the false positive rate (1-specificity) against the true positive rate (sensitivity). The area under the curve (AUC) can range from 0.5 (no discrimination) to 1.0 (perfect discrimination) and reflects the probability that in all possible pairs of workers in which one worker is sensitized and one is not, a higher predicted probability is assigned to the worker who is sensitized [Hanley and McNeil, 1982].

We used bootstrapping to assess the internal validity of the diagnostic model. This procedure provides an estimate of the optimism-corrected ROC area. The corrected estimate is the value that can be expected in future patients. We also obtained a shrinkage factor from bootstrap procedure. The regression coefficients were multiplied by this shrinkage factor to prevent that predictions are too optimistic for new patients, that is, low predictions are too low and high predictions too high [Harrell et al., 1996; Steyerberg et al., 2000]. External validity was studied in the British LA workers (validation set).

To facilitate application in practice, the regression coefficients of the diagnostic model were converted to a score chart. Then the sums of the scores were related to their corresponding probabilities. To derive the scores, the shrunk regression coefficients were divided by the smallest coefficient, and rounded to the nearest half integer. The sum scores were used to divide the population into a group of workers with a low and high risk of sensitization. Sensitivity and specificity of each score combination were plotted as ROC curves.

\section{Software}

All statistical analyses were performed with SPSS 10.0 for Windows (Statistical Products and Service Solution, Inc, Chicago) and S-plus version 4.5 software (Mathsoft, Inc., Seattle, WA).

\section{RESULTS}

\section{Worker Characteristics}

The general characteristics and univariable associations with sensitization to HMW allergens of workers in the development set are shown in Table I. Of 911 Dutch workers, $650(71 \%)$ were male and $146(16 \%)$ were sensitized to occupational HMW allergens. The mean age was 35 (SD 9.8) years, and the average of total working hours/week was 41 (SD 7.7).

\section{Model Development}

The continuous variable total working hours/week was transformed into a dichotomous variable. The RCS function showed a clear change in risk of sensitization at $38 \mathrm{hr} /$ week. The model $\chi^{2}$ of the RCS and dichotomization at $38 \mathrm{hr} /$ week yielded comparable results; $\chi^{2}=14.7$ for the continuous variable, and $\chi^{2}=11.8$ for dichotomous variable. Working more than $38 \mathrm{hr} /$ week significantly increased the risk of sensitization by almost two times $(\mathrm{OR}=1.9)$ compared to working less than $38 \mathrm{hr}$ (Table I).

We started the selection process with seven predictors, that were, gender, personal history of allergic symptoms, work-related allergic symptoms, total working hours/week, previous exposure to HMW allergens, IgE to common allergen, and total IgE level. In agreement with previous publications, age and smoking habits were not associated with sensitization (univariable $P$-value $>0.5$ ) and hence not considered in the backward selection [Cullinan et al., 1994a,b; Houba et al., 1996; Heederik et al., 1999]. Four predictors remained in the model after backward selection were work-related allergic symptoms, total working hours/ week, IgE to common allergen, and total IgE level.

Testing the interaction between the first four predictors and type of work gave a significant result $\left(\chi^{2}=11.6, \mathrm{df}=4\right.$, $P=0.021)$. Therefore, all interaction terms and type of work were included in the final model (Table II). 
TABLE I. General Characteristics and Univariate Association for Sensitization to Occupational HMW Allergens in Pooled Data (The Development Set) of Dutch Bakers and Laboratory Animal Workers

\begin{tabular}{lccc} 
& Distribution n (\%) & Univariable OR (95\% CI) & $P$-value \\
\hline Questionnaire & & & \\
Age $^{\mathrm{a}}$ & $35(9.7)$ & $0.99(0.98-1.01)$ & 0.508 \\
Male gender & $650(71)$ & $0.6(0.4-0.9)$ & 0.015 \\
Current smoker & $306(34)$ & $1.1(0.7-1.6)$ & 0.709 \\
Personal history of atopic symptoms & $301(33)$ & $1.9(1.3-2.8)$ & $<0.001$ \\
Work-related symptoms & $294(32)$ & $5.5(3.8-8.0)$ & $<0.001$ \\
Working $>38$ hr/week & $610(67)$ & $1.9(1.3-2.7)$ & 0.001 \\
Previous exposure to HMW allergens & $638(70)$ & $1.3(0.9-1.9)$ & 0.177 \\
$\quad$ Type of work (laboratoryanimal worker) & $568(52)$ & $1.0(0.7-1.4)$ & 0.996 \\
IgE results & & & $<0.001$ \\
Positive lgE to common allergens & $287(32)$ & $4.4(3.0-6.4)$ & $<0.001$ \\
$\quad$ Total lgE $\geq 100$ kU/L & $199(22)$ & $3.4(2.4-4.9)$ & \\
Outcome & & & \\
Sensitized to HMW allergens & $146(16)$ & & \\
\hline
\end{tabular}

$\mathrm{OR}$, odds ratio; $\mathrm{Cl}$, confidence interval.

${ }^{\mathrm{a}}$ Mean (standard deviation).

The interaction terms show that the strength for predictors for LA workers and bakers is different. For example, the interaction of type of work with work-related symptoms $(\mathrm{OR}=3.2)$ indicates that the strength of work-related symptom is 3.2 times higher in LA workers than in bakers. Hence, the OR for work-related symptoms is $6.7(2.1 * 3.2)$ in LA workers and 2.1 in bakers.

\section{Model Performance}

The calibration of the diagnostic model is given in Figure 1. Observed frequencies and predicted probabilities of sensitization were in agreement for all predicted probabilities categories. The Hosmer-Lemeshow goodness-of-fit test

TABLE II. Strength of the Predictors for Sensitization to Occupational HMW Allergens in the Model as Estimated in Pooled Data (The Development Set)

\begin{tabular}{|c|c|c|}
\hline & B (SE) & OR (95\% Cl) \\
\hline Work-related symptoms & $0.73(0.33)$ & $2.1(1.1-3.9)$ \\
\hline Working $>38 \mathrm{hr} /$ week & $0.87(0.32)$ & $2.4(1.3-4.5)$ \\
\hline Positive IgE to common allergens & $0.82(0.34)$ & $2.3(1.2-4.4)$ \\
\hline Total lgE level $\geq 100 \mathrm{kU} / \mathrm{L}$ & $0.59(0.35)$ & $1.8(0.9-3.6)$ \\
\hline Typeof work $^{\dagger}$ & $-0.68(0.50)$ & $0.6(0.2-1.3)$ \\
\hline Work-related symptoms*type of work & $1.17(0.43)$ & $3.2(1.4-7.4)$ \\
\hline Working $>38 \mathrm{hr} /$ week $^{*}$ type of work & $-0.33(0.48)$ & $0.7(0.3-1.8)$ \\
\hline Positive lgE to common allergens*type of work & $-0.43(0.44)$ & $0.6(0.3-1.5)$ \\
\hline Total $\lg \mathrm{E} \geq 100 \mathrm{kU} / \mathrm{L}^{*}$ type of work & $0.67(0.45)$ & $1.9(0.8-4.8)$ \\
\hline
\end{tabular}

$\mathrm{OR}$, odds ratio; $\mathrm{Cl}$, confidence interval.

Type of work: 1, laboratory animal workers; 0 , bakers.

${ }^{*}$ Single asterisk indicates interaction between variables. showed good agreement between the predicted and observed probabilities of sensitization $(P=0.2)$.

The discriminative ability of the diagnostic model in the development set was good (AUC 0.77; 95\% CI 0.73-0.82). The AUC estimated in laboratory in animal workers separately was 0.80 (95\% CI $0.75-0.86)$, whereas in bakers it was 0.70 (95\% CI 0.62-0.78). The model showed higher sensitivity with similar specificity in the LA workers, as compared to bakers, at all cut-off points (Fig. 2).

\section{Internal Validity}

The bootstrapping procedure yielded an optimismcorrected AUC of 0.76 and a shrinkage factor of 0.95. This result showed that the optimism was small (AUC difference was 0.01 , and the shrinkage factor of 0.95 was close to 1.0 ).

\section{Score Chart}

The diagnostic model was presented as a score chart. The scores differed for bakers and LA workers as result of the interaction with type of work (Table III). The sum scores correspond to predicted risks of being sensitized to occupational HMW allergens.

As shown, bakers and LA workers with two positive questionnaire predictors but negative serological test results had a sum score of $4.5(2.0+2.5+0$ for bakers and $5.0+1.5-2.0$ for LA workers). This corresponded to a probability of $23 \%$ of being sensitized. At this point, the sensitivity was $70 \%$ and specificity was $80 \%$ for LA workers, but $51 \%$ and $80 \%$ for bakers (Fig. 2). 
A

Calibration in Bakers Set

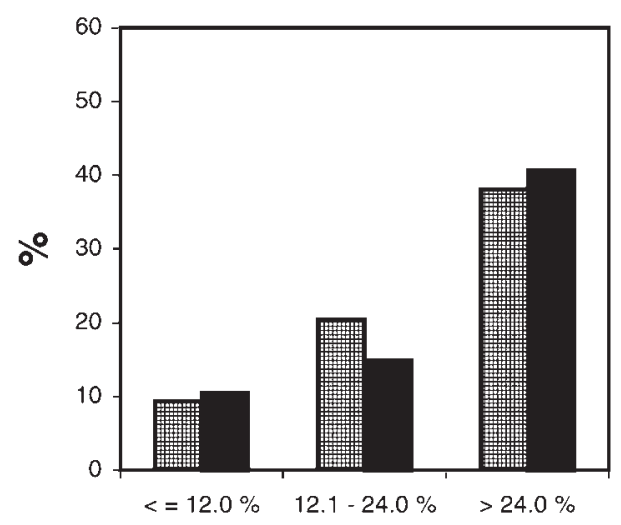

Average Predicted Probability OObserved proportion

B

Calibration in LA Set

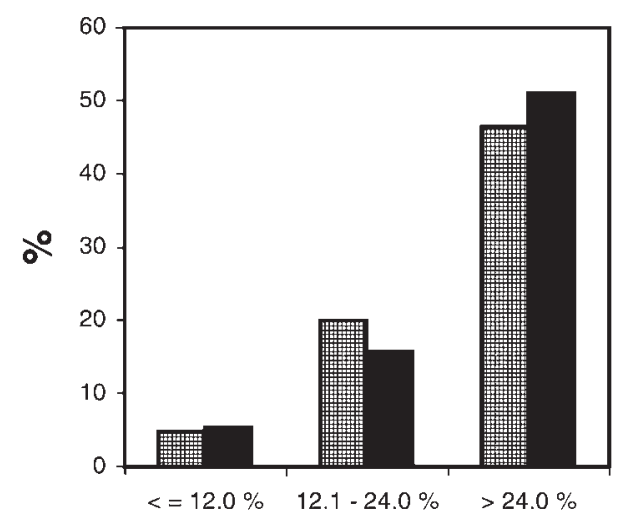

Average Predicted Probability D Observed proportion

C

Calibration in Validation Set

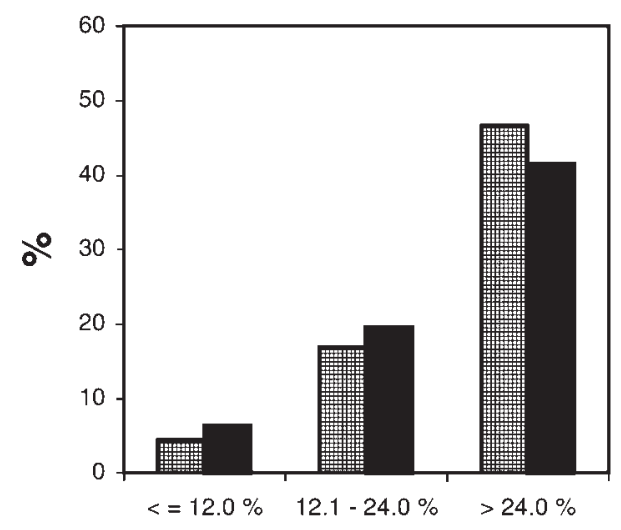

田Average Predicted Probability DObserved proportion

FIGURE 1. Agreement between the observed proportion and the average predicted probability of sensitization to occupational allergens in Dutch bakers and laboratory animal workers (the development set) and British laboratory animal workers (validationset).

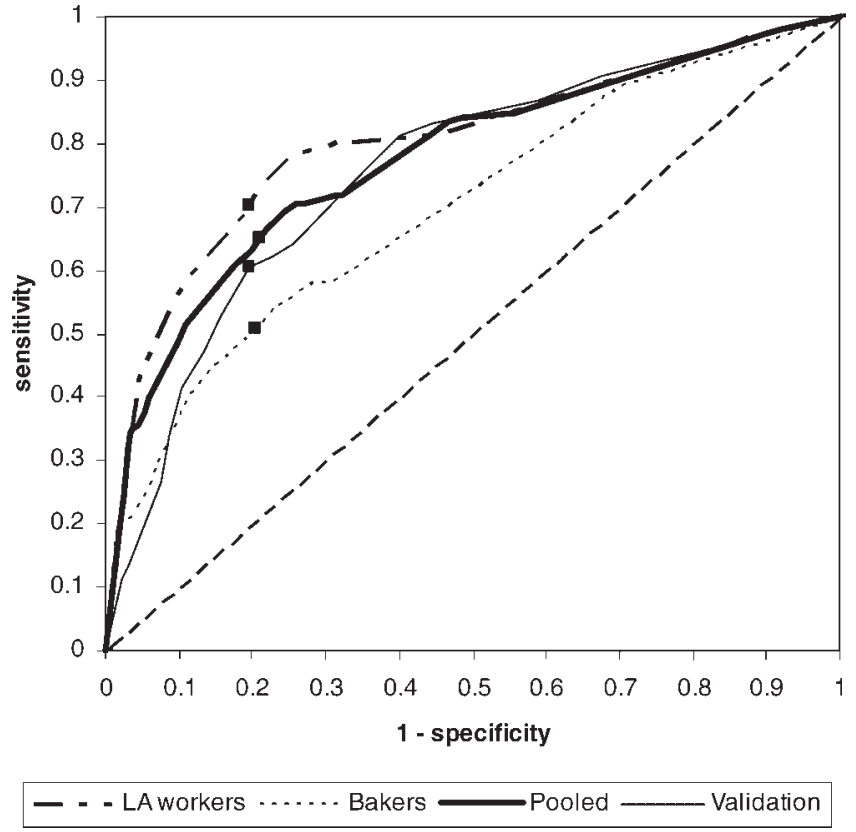

FIGURE 2. Discriminative ability of the score chart. At the same sum score of $4.5(\boldsymbol{\bullet})$, given similar specificity, the sensitivity was much lower in the bakers than in laboratory animal, whereas validation in British data showed in between sensitivity.

\section{External Validity}

Of 352 British LA workers 53 (15\%) were sensitized to HMW allergens. Workers in this validation set had shorter working hours/week (38.2 hr, SD 5.2), a lower proportion of workers had work-related allergic symptoms (24\%), but a higher proportion had elevated total IgE levels (42\%) than observed in the development set $(P<0.01)$.

The calibration of the diagnostic model is shown in Figure 1C. The Hosmer-Lemeshow goodness-of-fit test showed good agreement between the predicted probabilities and observed proportion of sensitization $(P=0.786)$. Figure 2 showed that the diagnostic model discriminated sensitized and non-sensitized workers reasonably well (AUC $0.76 ; 95 \%$ CI 0.68 to 0.83 )

\section{DISCUSSION}

Our study shows that it is possible to develop a generic diagnostic model to detect the presence of sensitization to occupational HMW allergens in LA workers and bakers. The same variables predicted sensitization in the two types of workers with different weighing of the strength of the predictors for different work environments. The model showed adequate external validity in an independent set of LA workers.

The model was easily applicable since it comprised four simple questionnaires and routine laboratory test predictors 


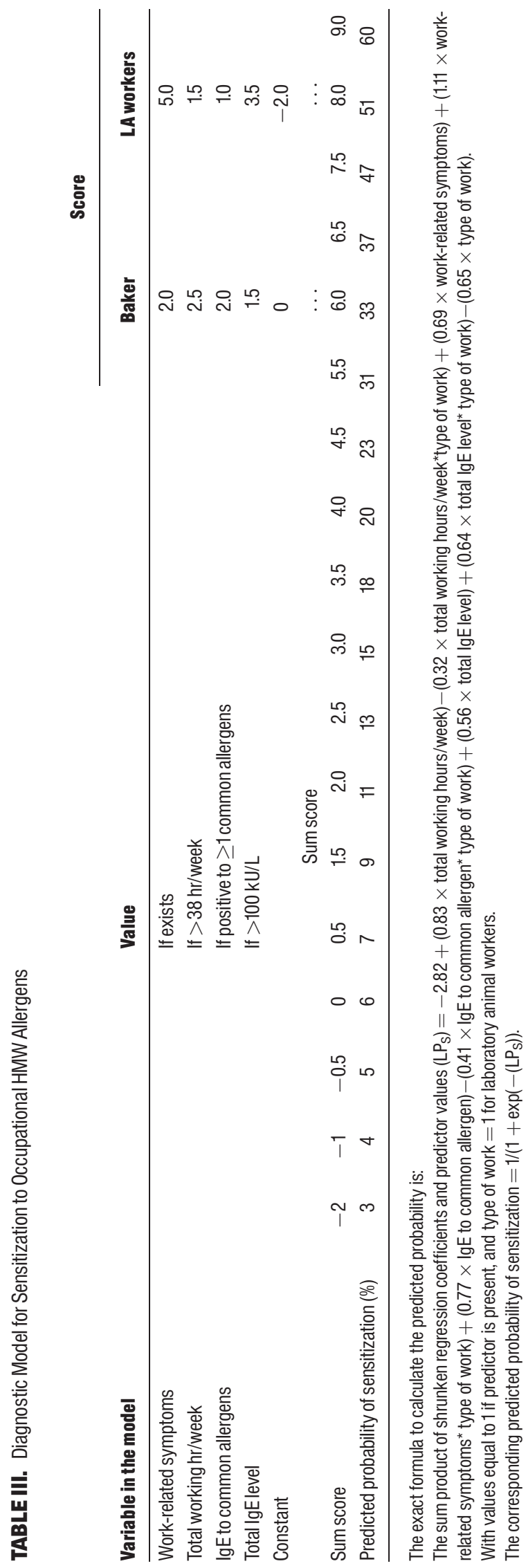

available in most occupational health practices. Our results showed that working $>38 \mathrm{hr} /$ week, presence of work-related allergic symptoms, total $\mathrm{IgE}>100 \mathrm{kU} / \mathrm{L}$ and positive $\mathrm{IgE}$ to common allergens were predictors for sensitization to occupational HMW allergens.

Interactions between type of work and work-related symptoms, total working hours, and the serologic tests showed that the strength of these variables in predicting the sensitization differed between bakers and LA workers. For instance, total working hours appeared to be a stronger predictor in bakery setting $(\mathrm{OR}=2.4)$ than in the LA setting $(\mathrm{OR}=2.4 * 0.7=1.7)$.

Several previous studies have shown that exposure level is a strong predictor of HMW allergens sensitization [Houba et al., 1998a; Jeffrey et al., 1999; Nieuwenhuijsen et al., 1999, 2003]. We did, intentionally, not include exposure level in our diagnostic model as we aimed to develop a model consisting of simple questionnaires and laboratory tests. Therefore, the total working hours may be considered as a simple proxy for cumulative exposure level. Further analyses indeed showed that total working hours differed across type of bakeries and job titles (tasks), and correlated with cumulative allergen exposure.

Another issue related to our modeling strategy concerns the preference of dichotomized variable instead of continuous variable. Dichotomization will somehow increase the possibility of misclassification for people who are just below or above the cut point. Though, we used dichotomized variable for reason of practical application. Further, the dichotomized variable fitted the data very similar to the RCS, because the increase in risk of sensitization was very steep at $38 \mathrm{hr}$.

Usually, sensitized workers are detected when they present themselves to the occupational physician because of symptoms. However, to find all sensitized workers the whole population must be studied in a survey-like approach that is less efficient and will result in high expenses.

In our approach, the diagnostic model serves as a triage system to split the population into high and low risk groups. The occupational physician could choose to follow only workers in the high risk group for sequential medical examination and occupational preventive intervention. In this way, the number of uninformative negative serological outcomes can be considerably limited.

An important step before using the diagnostic model, or score chart, is to determine the cut-off point of the sum score. In our example, we used a sum score of 4.5. At this point, the corresponding predicted probability was $23 \%$, which is higher than the prior probability $(16 \%)$. If we considered workers with a sum score of 4.5 or more at high risk, we could reduce the number of workers to be referred for further test to $27 \%$. However, at this point, 4 of 10 workers with sensitization will be missed (sensitivity 64\%). Increasing the cut-off level will further reduce the number of 
workers undergoing further tests but the number of false negative diagnosed workers will similarly increase.

Recently, another diagnostic model was developed in only Dutch LA workers [Meijer et al., 2004]. The model consisted of only questionnaire variables, specifically for LA workers that do not apply to bakers. Consequently, its use is limited to workers exposed to LA allergens.

In conclusion, regardless of different weights of predictors across type of work, we can use the same variables to predict sensitization in bakers and LA workers. Our diagnostic model proved to be reliable and discriminated well between workers with high and low risk of sensitization of HMW allergens. Satisfactory validation in 352 British LA workers showed a good transportability in different geographic area.

\section{REFERENCES}

Cullinan P, Lowson D, Nieuwenhuijsen MJ, Gordon S, Tee RD, Venables KM, McDonald JC, Newman Taylor AJ. 1994a. Work related symptoms, sensitisation, and estimated exposure in workers not previously exposed to laboratory rats. Occup Environ Med 51:589592.

Cullinan P, Lowson D, Nieuwenhuijsen MJ, Sandiford C, Tee RD, Venables KM, McDonald JC, Newman Taylor AJ. 1994b. Work related symptoms, sensitisation, and estimated exposure in workers not previously exposed to flour. Occup Environ Med 51:579-583.

Doekes G, Douwes J, Wouters I, de Wind S, Houba R, Hollander A. 1996. Enzyme immunoassays for total and allergen specific $\operatorname{IgE}$ in population studies. Occup Environ Med 53:63-70.

Hanley JA, McNeil BJ. 1982. The meaning and use of the area under a receiver operating characteristic (ROC) curve. Radiology 143:29-36.

Harrell FE, Jr., Lee KL, Mark DB. 1996. Multivariable prognostic models: Issues in developing models, evaluating assumptions and adequacy, and measuring and reducing errors. Stat Med 15:361-387.
Harrell Frank E. 2001. Regression modeling strategies: With applications to linear models, logistic regression, and survival analysis. New York, London Springer. 81p.

Heederik D, Venables KM, Malmberg P, Hollander A, Karlsson AS, Renstrom A, Doekes G, Nieuwenhijsen M, Gordon S. 1999. Exposureresponse relationships for work-related sensitization in workers exposed to rat urinary allergens: Results from a pooled study. J Allergy Clin Immunol 103:678-684.

Hollander A, Doekes G, Heederik D. 1996. Cat and dog allergy and total IgE as risk factors of laboratory animal allergy. J Allergy Clin Immuno 98:545-554.

Houba R, Heederik DJ, Doekes G, van Run PE. 1996. Exposuresensitization relationship for alpha-amylase allergens in the baking industry. Am J Respir Crit Care Med 154:130-136.

Houba R, Doekes G, Heederik D. 1998a. Occupational respiratory allergy in bakery workers: A review of the literature. Am J Ind Med 34:529-546.

Houba R, Heederik D, Doekes G. 1998b. Wheat sensitization and workrelated symptoms in the baking industry are preventable. An epidemiologic study. Am J Respir Crit Care Med 158:1499-1503.

Jeffrey P, Griffin P, Gibson M, Curran AD. 1999. Small bakeries-A cross-sectional study of respiratory symptoms, sensitization and dust exposure. Occup Med (Lond) 49:237-241.

Meijer E, Grobbee DE, Heederik D. 2004. A strategy for health surveillance in laboratory animal workers exposed to high molecular weight allergens. Occup Environ Med 61:831-837.

Nieuwenhuijsen MJ, Heederik D, Doekes G, Venables KM, Newman Taylor AJ. 1999. Exposure-response relations of alpha-amylase sensitisation in British bakeries and flour mills. Occup Environ Med 56:197-201.

Nieuwenhuijsen MJ, Putcha V, Gordon S, Heederik D, Venables KM, Cullinan P, Newman-Taylor AJ. 2003. Exposure-response relations among laboratory animal workers exposed to rats. Occup Environ Med 60:104-108.

Steyerberg EW, Eijkemans MJ, Harrell FE, Jr., Habbema JD. 2000 Prognostic modelling with logistic regression analysis: A comparison of selection and estimation methods in small data sets. Stat Med 19:10591079. 